\title{
Perioperative immunotherapy for muscle-invasive bladder cancer
}

\author{
In-Ho Kim ${ }^{1}$, Hyo Jin Lee ${ }^{2}$ \\ ${ }^{1}$ Department of Internal Medicine, Division of Medical Oncology, Seoul St. Mary's Hospital, The Catholic University of Korea College of Medicine, \\ Seoul, Korea; ${ }^{2}$ Department of Internal Medicine, Chungnam National University School of Medicine, Daejeon, Republic of Korea \\ Contributions: (I) Conception and design: All authors; (II) Administrative support: All authors; (III) Provision of study materials or patients: All \\ authors; (IV) Collection and assembly of data: All authors; (V) Data analysis and interpretation: All authors; (VI) Manuscript writing: All authors; (VII) \\ Final approval of manuscript: All authors. \\ Correspondence to: Hyo Jin Lee. Department of Internal Medicine, Chungnam National University School of Medicine, Daejeon 35015, Republic of \\ Korea. Email: cymed@cnuh.co.kr.
}

\begin{abstract}
Radical cystectomy is the standard of care treatment for patients with localized muscleinvasive bladder cancer (MIBC). However, patients with MIBC experience high rates of relapse despite primary therapy, and perioperative strategy is an important treatment option. Cisplatin-based neoadjuvant chemotherapy was associated with improved prognosis, and adjuvant chemotherapy is also an important option for selected patients. However, perioperative chemotherapy is not effective in some patients. Moreover, the currently recommended perioperative treatment is cisplatin-based chemotherapy; approximately $50 \%$ of the patients are ineligilble for cisplatin treatment owing to various reasons such as medical comorbidities, poor performance status, and renal insufficiency. The recent success of treatment with immune checkpoint inhibitors (ICIs) suggests that ICIs is the new standard therapy for patients with metastatic bladder cancer. Furthermore, ICIs showed more favorable toxicity profiles than conventional cytotoxic chemotherapy. These results indicate that ICIs may play a role in the treatment of muscle-invasive disease, and many recent studies have been conducted in a perioperative setting. The present review aims to summarize and discuss the current perioperative strategy of immunotherapy focused on ICIs based on recent ongoing clinical trials.
\end{abstract}

Keywords: Bladder cancer; immunotherapy; perioperative systemic treatment

Submitted Sep 28, 2019. Accepted for publication Nov 20, 2019.

doi: $10.21037 /$ tau.2019.11.31

View this article at: http://dx.doi.org/10.21037/tau.2019.11.31

\section{Introduction}

Bladder cancer is the $12^{\text {th }}$ most common malignancy worldwide, with an estimated 200,000 deaths from this disease every year (1). Owing to early micrometastatic dissemination, patients with muscle-invasive bladder cancer (MIBC) and those with upper tract urothelial carcinoma (UC) experience high rates of relapse despite primary therapy. MIBC has a high metastatic potential at diagnosis but is still often curable with aggressive management. According to current treatment guidelines, cisplatin-based neoadjuvant chemotherapy (NAC) is recommended for patients with cT2-4aN0M0 MIBC, and adjuvant cisplatinbased chemotherapy is selectively recommended for patients with locally advanced (pT3/4) and/or lymph node- positive disease $(2,3)$. Although the currently recommended perioperative treatment is cisplatin-based chemotherapy, approximately $50 \%$ of the patients are ineligilble for cisplatin treatment owing to various reasons such as poor performance status, medical comorbidity, and renal insufficiency (4). These patient populations represent those patients in whom there is no effective systemic therapy for treating micrometastatic spread.

To date, for various types of cancers, including bladder cancer, several immunotherapeutic agents-which block immune checkpoints-have been investigated and/or clinically used, such as nivolumab and pembrolizumab that block programmed cell death receptor 1 (PD-1); atezolizumab, durvalumab, and avelumab that block PD- 
Table 1 Summary of immune checkpoint inhibitors in metastatic UC



UC, urothelial carcinoma; ORR, objective response rate; OS, overall survival; PFS, progression free survival; TRAE, treatment related adverse event; DLT, dose limiting toxicity.

ligand-1 (PD-L1); and ipilimumab and tremelimumab that block cytotoxic $\mathrm{T}$ lymphocyte-associated protein 4 (CTLA-4). Among these, the immune checkpoint inhibitors (ICIs) of atezolizumab, pembrolizumab, nivolumab, durvalumab, and avelumab have already been approved for clinical use in bladder cancer by the United States Food and Drug Administration (5). These results suggest that ICIs may have a role in the perioperative setting of MIBC, which has recently led to several clinical trials.

Herein, we briefly review immunotherapy that is currently being implemented in the perioperative setting, focusing on ICIs that have been extensively evaluated for MIBC, and discuss the clinical implications of this treatment.

\section{Data searching process}

A literature search for current data on perioperative ICIs was conducted using PubMed and ClinicalTrials. gov databases. We searched data using the following combinations of MeSH terms: "urothelial carcinoma" OR "bladder cancer" and "immunotherapy" OR "atezolizumab" OR "nivolumab" OR "ipilimumab" OR "pembrolizumab" OR "avelumab" OR "durvalumab" OR "tremelimumab" and "neoadjuvant" OR "adjuvant" OR "preoperative" OR "postoperative" OR "perioperative." Prospective and retrospective studies were included. In addition, as immunotherapy is a rapidly developing field, we examined abstracts from major oncology conferences between 2012 and 2019. Moreover, because many perioperative ICI trials are currently ongoing, these clinical trials were included (Clinicaltrials.gov).

\section{Neoadjuvant immunotherapy}

Cisplatin-based NAC followed by surgery is the standard of care for MIBC. A previous study showed that NAC with methotrexate, vinblastine, doxorubicin, and cisplatin followed by radical cystectomy was associated with longer survival of patients with MIBC and without any residual cancer in surgical specimens (6). In another study, NAC with cisplatin, methotrexate, and vinblastine resulted in a significant survival benefit compared with surgery and/or radiotherapy alone in patients with MIBC (7). Cisplatinbased NAC was associated with a pathologic complete response (pCR) rate of $30-40 \%$ and a $5 \%$ improvement in overall survival (OS) $(3,8)$. However, NAC is performed for only $10-20 \%$ of patients, and approximately $50 \%$ of patients are ineligible to receive cisplatin; therefore, as it is difficult to administer NAC to all patients, a new treatment strategy is needed (9).

The recent success of treatment with ICIs for UC suggests that ICI is the new standard therapy for UC, especially metastatic UC (Table 1). In an open-label, international, phase 3 trial, pembrolizumab was associated with improved OS and low rates of treatment-related adverse events in patients with platinum-refractory advanced UC (13). In the openlabel, multicenter, single-arm, phase 2 IMvigor 210 trial (12), patients treated with atezolizumab showed favorable response rates, survival, and tolerability. In the Checkmate 275 study (14), multicenter, single-arm, phase 2 trial, nivolumab was associated with favorable clinical benefit and manageable toxicity in previously treated patients with locally advanced or metastatic UC. Durvalumab and avelumab also showed similar results $(15,16)$. These studies showed an objective response rate of approximately $20 \%$, and the incidence of 
Table 2 Summary of clinical trials of neoadjuvant immunotherapy in MIBC

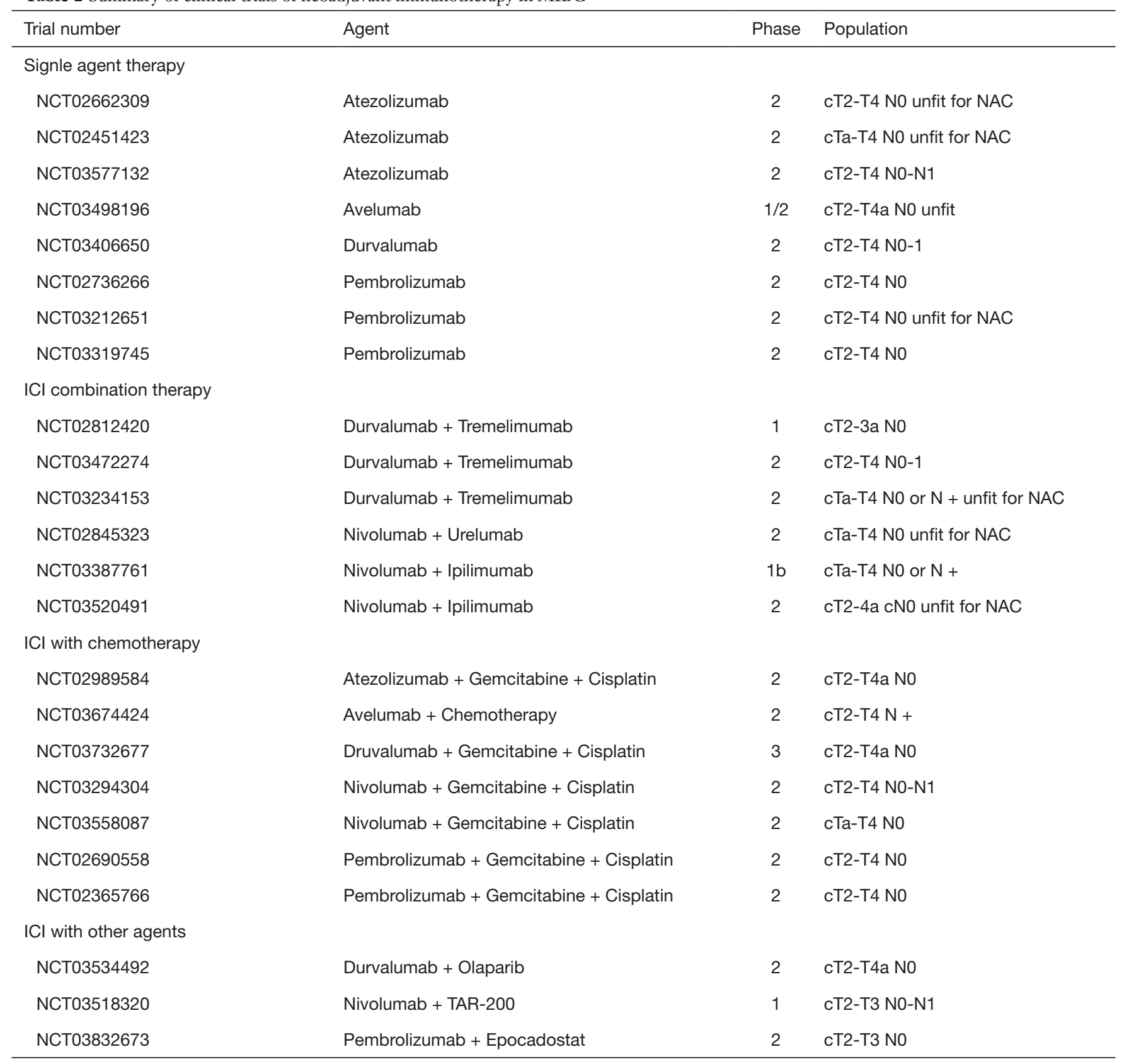

MIBC, muscle-invasive bladder cancer; NAC, neoadjuvant chemotherapy; ICl, immune checkpoint inhibitor.

treatment-related grade $\geq 3$ adverse events was approximately $6-18 \%$. Moreover, several clinical trials evaluated the use of ICIs as first-line treatment for patients who are ineligible for cisplatin treatment. Keynote-052 trial, a multicenter, singlearm, phase 2 study, showed that pembrolizumab has antitumor activity and acceptable tolerability in patients with UC who are ineligible for cisplatin treatment (11). In another single-arm, multicenter, phase 2 study, atezolizumab showed encouraging response rates, survival, and tolerability, supporting its therapeutic use for untreated metastatic UC (10).

Owing to the success of ICIs for treating metastatic cancer, many studies have recently evaluated the efficacy of neoadjuvant ICIs for MIBC (Table 2). The recently 
published phase 2 PURE-01 trial was designed to assess the efficacy of single-agent, neoadjuvant pembrolizumab for MIBC, in addition to determining the biomarkers (17). Patients were enrolled regardless of their cisplatin eligibility and received three cycles of $200 \mathrm{mg}$ pembrolizumab every 3 weeks before radical cystectomy. The primary endpoint was the pCR. Among the 50 enrolled patients, 27 (54\%) had clinical (c)T3 disease, 21 (42\%) had cT2 disease, and $2(4 \%)$ had cT2-3N1 disease. All the patients treated with pembrolizumab underwent radical cystectomy. A pCR and downstaging to non-muscle-invasive tumors were observed in $42 \%$ and $54 \%$ of patients, respectively. A total of $54.3 \%$ of the patients with PD-L1 combined positive score (CPS) $\geq 10$ showed pCR, whereas only $13.3 \%$ of patients with CPS $<10$ showed pCR. A significant nonlinear association was observed between pCR and tumor mutation burden, with a cutoff at 15 mutations/Mb. Additionally, pembrolizumab was associated with few immune-related adverse events and did not delay planned surgery, and postsurgical complications were consistent with those observed in the most recent literature about open and robot-assisted procedures (18).

The ABACUS trial (19), a single-arm phase 2 study, investigated the efficacy and safety of 2 cycles of atezolizumab (1,200 $\mathrm{mg}$ q3weeks) prior to cystectomy for MIBC (T2-4N0M0). In total, 69 patients who refused or were ineligible for cisplatin-based NAC were included. The pCR rate was $29 \%$, and $39 \%$ of cases were downstaged to non-muscle-invasive disease. A total of $40 \%$ of patients with PD-L1-positive disease $(\geq 5 \%$ immune cells with SP142 antibody) showed pCR, whereas $10 \%$ of those with PD-L1-negative disease showed pCR. Moreover, sequential biomarker analysis showed an increase in PDL1 and CD8 expression after treatment with atezolizumab. Only one patient experienced significant progression during neoadjuvant atezolizumab treatment. Neoadjuvant atezolizumab treatment was also safe and feasible. Surgical complications (Clavien-Dindo grade 3 or 4 ) were observed in $10 \%$ of patients.

Recent trends in immunotherapy include combination treatment of ICIs with other agents such as cytotoxic agents, other immunotherapy, and target agents, as evaluated in small prospective studies. Hoimes et al. presented the results of a phase Ib/II study of neoadjuvant pembrolizumab and chemotherapy for MIBC at ESMO 2018 (20). Patients with cT2-4aN0M0 UC or mixed histology were included in cohort I (cisplatin-eligible) or cohort II (cisplatin-ineligible). Pembrolizumab $200 \mathrm{mg}$ q3 weeks was administered on day
8 for five doses, with cisplatin $\left(70 \mathrm{mg} / \mathrm{m}^{2}\right)$ on day 1 and gemcitabine $\left(1,000 \mathrm{mg} / \mathrm{m}^{2}\right)$ on days 1 and 8 of a 21 -day cycle, for four cycles, followed by radical cystectomy with node dissection. The median number of doses of pembrolizumab, cisplatin, and gemcitabine was 5, 4, and 8, respectively. During phase $\mathrm{Ib}$ of the study, no dose-limiting toxicities were observed in six patients. Grade 3/4 cytopenia was observed in $57 \%$ of patients. One patient did not undergo surgery due to grade 4 thrombocytopenic purpura. The rate of pathologic downstaging was $60 \%$, and it was not correlated with the baseline PD-L1 level.

The pilot results of durvalumab with tremelimumab (anti-CTLA-4) in patients with high-risk MIBC who were ineligible for cisplatin-based NAC were presented in ASCO 2019 (21). In this single-arm study, neoadjuvant durvalumab with tremelimumab was administered to patients with localized, high-risk MIBC (cT2-T4a) who were ineligible for cisplatin-based NAC. Among 28 enrolled patients, 21 patients have completed cystectomy. Of the 21 included patients, 9 (43\%) had pCR, and 14 (67\%) showed downstaging of disease. Of 28 patients, 5 (17\%) developed grade 3 immune-related toxicity, and $2(7 \%)$ experienced a delay in surgery of $>30$ days. Immune profiling of baseline peripheral blood indicates that patients with pCR have a significantly lower frequency of a Th2 subset than patients with upstaging of disease. In addition, gene expression profiling analysis of baseline tumor tissues revealed a significantly less immunosuppressive microenvironment in patients with pCR than in patients with upstaging of disease.

A NEODURVARIB trial, open-label phase II singlearm trial about the combination of durvalumab and olaparib prior to surgery for resectable MIBC, was presented in ASCO 2019 (22). The primary endpoint is to assess the impact of neoadjuvant treatment with durvalumab plus olaparib in the molecular profile of MIBC. Durvalumab $1,500 \mathrm{mg} \mathrm{q} 4$ weeks and olaparib $150 \mathrm{mg}$ bid orally from 6 to a maximum of 8 weeks pre-cystectomy was administered. This trial is ongoing, and the result is not yet reported.

\section{Adjuvant immunotherapy}

The role of adjuvant chemotherapy for bladder cancer is still unclear. Although several studies have been conducted regarding the use of adjuvant chemotherapy for bladder cancer, the trials failed or were terminated prematurely owing to poor study design and limited patient recruitment $(23,24)$. The largest trial $(25)$ that compared adjuvant cisplatin-based combination chemotherapy after radical 
Table 3 Summary of clinical trials of adjuvant immunotherapy in MIBC

\begin{tabular}{|c|c|c|c|c|c|c|c|c|c|c|}
\hline Study & Study ID & Phase & Agent & Control & $\mathrm{N}$ & $\begin{array}{l}\text { Primary } \\
\text { endpoint }\end{array}$ & $\begin{array}{l}\text { Secondary } \\
\text { endpoint }\end{array}$ & $\begin{array}{l}\text { Upper } \\
\text { tract }\end{array}$ & $\begin{array}{c}\text { Cisplatin } \\
\text { based } \\
\text { NAC }\end{array}$ & $\begin{array}{c}\text { Estimated } \\
\text { primary } \\
\text { completion date }\end{array}$ \\
\hline Imvigor010 & NCT02450331 & 3 & Atezolizumab & Observation & 809 & DFS & $\begin{array}{l}\text { (I) OS; (II) } \\
\text { DSS; (III) } \\
\text { DMFS; (IV) } \\
\text { NUTRFS }\end{array}$ & Included & Included & January, 2020 \\
\hline
\end{tabular}

MIBC, muscle-invasive bladder cancer; NAC, neoadjuvant chemotherapy; OS, overall survival; DFS, disease free survival; PD-L1, programmed death ligand 1; NUTRFS, non-urothelial track recurrence free survival; DSS, disease specific survival; DMFS, distant metastasis free survival.

cystectomy with deferred chemotherapy for pathologic (p)T3-pT4 or N + M0 UC of the bladder showed longer progression-free survival but no significant improvement in OS. In 2010, a Spanish group published the results of a preliminary study that compared adjuvant paclitaxel, gemcitabine, and cisplatin with observation for resectable high-risk MIBC (23). This study was prematurely terminated owing to poor recruitment, with 142 patients randomized ( 74 to the observation am and 68 to the adjuvant chemotherapy arm). In that study, OS and diseasefree survival (DFS) were significantly more prolonged in the adjuvant chemotherapy arm than in the observation arm. However, as the study was prematurely terminated, the results were very limited.

Another trial was performed by an Italian group (24). This study evaluated the benefit of adjuvant gemcitabine with cisplatin versus surgery alone in patients with MIBC. This study did not show any improvement in the OS and DFS of the adjuvant arm compared to the observation arm. However, as only 200 patients were actually registered in the clinical trial instead of the planned 600 patients, the clinical implications were limited.

Adjuvant chemotherapy has some limitations in clinical fields. Many patients who underwent surgery are more likely to be ineligible for adjuvant cisplatin-based chemotherapy owing to impaired renal function or poor performance status after surgery. Moreover, a substantial number of patients received NAC before surgery but still had persistent muscle-invasive disease. In such cases, sequential postoperative cisplatin-based chemotherapy is often impossible.

ICI results in fewer toxicities than conventional cytotoxic chemotherapy as well as low kidney metabolization, which is an advantage in patients with decreased renal function. Moreover, considering the benefits currently shown for metastatic disease, adjuvant immunotherapy can be another option instead of adjuvant chemotherapy.

Three major phase 3 trials about adjuvant ICIs are currently ongoing (Table 3). The results of the three studies have not been reported to date. These studies included patients who did not receive NAC as well as those who had persistent muscle-invasive disease after NAC. Moreover, these trials are unique because they include patients with upper-tract UC. These trials are all about single ICI agent.

\section{Neoadjuvant versus adjuvant immunotherapy}

To date, there is no consensus on whether neoadjuvant or adjuvant immunotherapy is appropriate. Neoadjuvant treatment has several advantages (26). First, it enables response monitoring and provides prognostic information. In addition, neoadjuvant treatment downstages the tumor, allowing for less extensive surgery, thereby reducing postoperative complications. Finally, it uses pCR as a surrogate marker of recurrence-free survival and OS. Moreover, NAC might be better tolerated than adjuvant treatment following surgery, owing to the relevant postsurgery morbidity that might prevent reasonable adjuvant treatment $(27,28)$.

The advantages of neoadjuvant immunotherapy may 


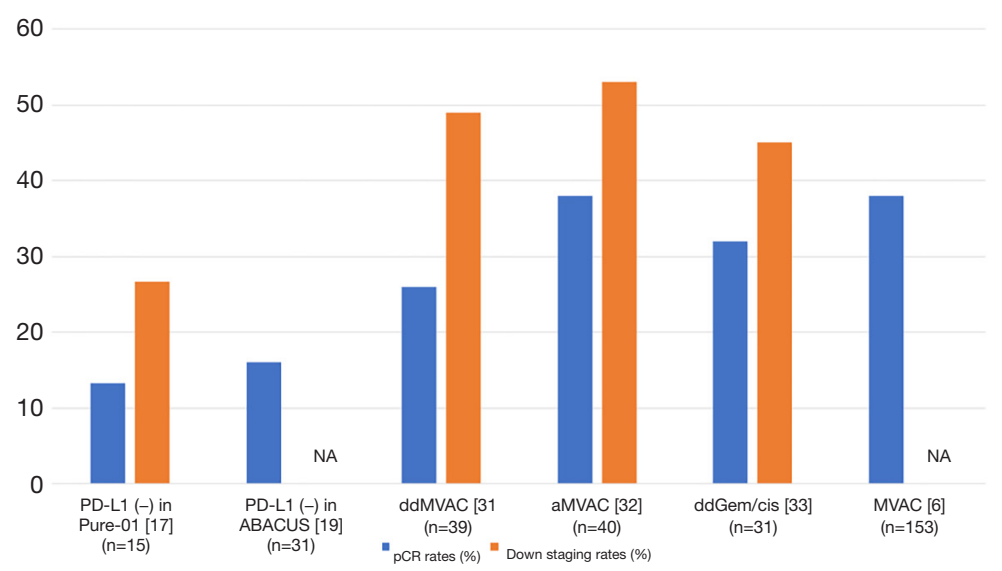

Figure 1 Summary of rates of pCR and downstaging in neoadjuvant chemotherapy and immunotherapy. PD-L1, programmed death ligand 1; NA, data not shown in reference; ddMVAC, dose dense MVAC; aMVAC, accelerated MVAC; ddGEM/Cis, dose dense Gem/cis.

be owing to the hypotheses explained below. Tumorinfiltrating lymphocytes are most often the cells that express the targets for ICIs, and there is an abundance of tumor antigens available for cross-priming at the time of immunotherapy (29). A previous pre-clinical study (30) showed the higher therapeutic efficacy of neoadjuvant than adjuvant immunotherapy to eradicate distant metastases after tumor resection. These hypotheses suggest that immunotherapy may be more likely to be appropriate in the neoadjuvant setting than in the adjuvant setting. However, the major challenge for using a single ICI is the short window of opportunity before surgery that maintains the chance of achieving a cure without delaying the timing for optimal surgery. According to the Keynote 052 trial (11), in untreated patients with metastatic disease, the objective response rate after single ICI was $20 \%$, and approximately $40 \%$ of patients showed progressive disease as the best response. Therefore, unresponsiveness to neoadjuvant ICI alone can be a major hurdle because it can lead to treatment failure. Accordingly, biomarker-driven neoadjuvant ICI may be necessary in the future. The abovementioned ABACUS (19) and PURE-01 (17) studies about neoadjuvant ICI showed improved response rates in the PD-L1-positive group. However, the pCR and downstaging rates were lower in the $\mathrm{PD}-\mathrm{L} 1-$-negative patients than in the patients who were previously treated with cisplatinbased NAC (Figure 1) $(6,17,19,31-33)$. Another important challenge associated with the use of neoadjuvant ICIs is the occurrence of perioperative complications. This is in line with the fact that neoadjuvant "cytotoxic chemotherapy" is currently a standard treatment, but it is not often performed owing to the possibility of perioperative complications.

To date, there is no study which has focused on the perioperative complications that may be associated with neoadjuvant ICIs in patients with MIBC. The RAZOR study compared the efficacy of robot-assisted cystectomy and open radical cystectomy, and showed that robotic cystectomy was non-inferior to open cystectomy considering the 2-year progression-free survival (18). In this study, the surgical complications were evaluated on the basis of the ClavienDindo classification system, and there is no significant difference in the incidence of surgical complications between the robotic and open cystectomy approaches. Comparing indirectly these results with the perioperative complications observed in the ABACUS (19) and PURE-01 (17) trials, there was no numerically significant difference between neoadjuvant ICIs and surgery without neoadjuvant ICI (Table 4). This finding suggests that the effect of neoadjuvant ICIs on perioperative complication may not be different when surgery is performed without neoadjuvant ICI. Nevertheless, as these comparisons are not performed directly, further studies should be performed. Moreover, treatment-related adverse events (TRAEs) from ICIs should be considered before surgery. Recently, many studies evaluating the use of ICIs for many cancers have shown the occurrence of TRAEs owing to ICIs. TRAEs due to ICIs for UC have been estimated in several previous studies on ICIs for metastatic disease, and the incidence of grade 3 or higher TRAEs was approximately $15 \%(10-13,34)$.

In the ABACUS (19) and PURE-01 (17) studies, the incidence of grade 3 or higher TRAEs of ICIs was $11 \%$ and $6 \%$ respectively, which are lower numerically than the 
Table 4 Summary of perioperative complication in surgery with or without neoadjuvant ICI

\begin{tabular}{lcccc}
\hline \multirow{2}{*}{ Perioperative complication } & \multicolumn{3}{c}{ Study } \\
\cline { 2 - 5 } & ABACUS (19) & PURE-01 (17) & RAZOR-robot (18) & RAZOR-open (18) \\
\hline Grade 0 (\%) & 48 & 50 & 33 & 31 \\
Grade 1-2 (\%) & 45 & 20 & 45 & 47 \\
Grade 3-4 (\%) & 17 & 30 & 19 & 19 \\
\hline
\end{tabular}

Perioperative complication was assessed using the Clavien-Dindo classification system grade. ICI, immune checkpoint inhibitor.

incidence of TRAEs when ICIs were used to treat metastatic disease. In the PURE-01 (17) study, the most frequent all-grade $\mathrm{AE}$ was thyroid dysfunction (18\%), and there were three patients $(6 \%)$ with grade 3 TRAEs that caused pembrolizumab discontinuation. There were a few delayed immune-related AEs, including pyrexia (6\%), pruritus (6\%), and xerostomia (4\%). All of the latter AEs occurred within 2 months postoperatively, and three patients required corticosteroid treatment. It is impossible to compare these results directly. But considering that ICI were administered for relatively short courses to MIBC patients in neoadjuvant setting than metastatic disease, it can be inferred that the incidence of TRAEs owing to neoadjuvant ICIs may not differ significantly from that observed in other studies that were performed for metastatic disease. Further studies should be performed. Furthermore, the above-mentioned studies [i.e., the ABACUS (19) and PURE-01 (17) trials] are on single ICI, and future studies on combination strategies will show different toxicity profiles; accordingly, careful observation and management of TRAEs will be required before performing the surgical approach.

Adjuvant treatment with ICIs has some advantages, including not delaying the timing of definitive local treatment. It can also reduce the incidence of postoperative complications owing to neoadjuvant ICIs. Moreover, in some cases, the pathological stage is higher than the preoperative stage, thereby resulting in the need for adjuvant strategy. Accordingly, adjuvant immunotherapy may also be an important option for selected patients.

\section{Predictive biomarkers for perioperative immunotherapy}

Currently, there are no standard predictive biomarkers of ICI in the perioperative setting as well for metastatic disease. Therefore, we discuss some potential biomarkers that indirectly have been studied in trials in metastatic disease.

Although PD-L1 expression has been studied in many clinical trials, there has been no conclusion till date regarding its use as a predictive biomarker. Previous studies regarding metastatic disease showed different results in the prognosis according to PD-L1 expression (10-14,34,35). These different results may be due to the diversity of the methods currently used for scoring PD-L1, the different cutoff values of PD-L1 expression, intratumoral/ intertumoral heterogeneity, and dynamic changes in PD-L1 expression (36). Moreover, because up to $10 \%$ of cases with low PD-L1 expression can show response to ICI, PD-L1 expression alone is very limited as a predictive biomarker.

The mutation burden has also extensively studied recently. Cancers with higher rates of somatic mutations showed better responses to immunotherapy (37-39). In both the cohorts in the IMvigor $210 \mathrm{UC}$ trial, a higher mutational load was correlated with a greater response to and longer OS after atezolizumab, and these associations were independent of PD-L1 expression $(10,12)$. However, more research is needed to determine whether the response can be accurately predicted by using only the tumor mutation burden. A recent study suggested the heterogeneity of tumor antigens and tumor mutation burden as biomarkers (40).

Gene expression (mRNA) subtype was also studied as a biomarker. TCGA analysis of RNA-seq data from 129 tumors identified four clusters (clusters I-IV) as biomarkers (41). Cluster I (papillary-like) was enriched in tumors with papillary morphology and FGFR3 alteration. Clusters I and II are similar to luminal A breast cancer, with the expression of urothelial markers such as GATA3. Cluster III is similar to basal-like breast cancer and head and neck squared cell carcinoma. Cluster IV is similar to cluster III, but with features of the surrounding stroma and muscle. The IMvigor 210 cohort 2 trial showed that the response rates to atezolizumab were significantly 
higher in the luminal cluster II subtype than in the other subtypes (12). In contrast, cluster III showed the highest response rates to nivolumab in the Checkmate 275 trial (14). However, the gene expression data of those two trials are not publicly available, and the methodology for assigning TCGA clusters was not revealed. Therefore, the results should be interpreted cautiously.

Although recent research indicated several potential biomarkers such as DNA damage repair and immune gene signatures, these biomarker studies were performed in the metastatic setting; the pathophysiology differs between the localized and metastatic settings. Therefore, the results of large-scale phase 3 studies that are ongoing or scheduled in the future in the perioperative setting need to be verified.

\section{Conclusions}

The introduction of ICIs changed the treatment landscapes for bladder cancer. In particular, ICIs became a new standard treatment option for patients with metastatic disease who are refractory or ineligible to platinum, owing to the better survival benefits and relatively low toxicity compared to other conventional cytotoxic chemotherapy. These results indicate that ICIs may play a role in the treatment of muscle-invasive disease, with many recent studies conducted in the perioperative setting.

Till date, there is no consensus on whether neoadjuvant or adjuvant immunotherapy is appropriate, and it is not clear whether a single ICI or ICIs in combination with other agents is more appropriate. In addition, as treatment in the perioperative setting increases the curative rate, various parameters should be considered, such as the medical condition, optimal surgery timing, and predictive biomarkers, while selecting the treatment strategy. To date, many trials are currently ongoing, and most of the results have not yet been reported. We hope that the results of these trials will provide clarifications about the abovementioned issues.

\section{Acknowledgments}

Funding: This work was supported by grant from the National Research Foundation of Korea (NRF2017R1A5A2015385).

\section{Footnote}

Provenance and Peer Review: This article was commissioned by the Guest Editors (Ja Hyeon Ku, Ho Kyung Seo, Seok Ho Kang) for the series "Muscle-Invasive Bladder Cancer" published in Translational Andrology and Urology. The article has undergone external peer review.

Conflicts of Interest: Both authors have completed the ICMJE uniform disclosure form (available at http://dx.doi. org/10.21037/tau.2019.11.31). The series "Muscle-Invasive Bladder Cancer" was commissioned by the editorial office without any funding or sponsorship. The authors have no other conflicts of interest to declare.

Ethical Statement: The authors are accountable for all aspects of the work in ensuring that questions related to the accuracy or integrity of any part of the work are appropriately investigated and resolved.

Open Access Statement: This is an Open Access article distributed in accordance with the Creative Commons Attribution-NonCommercial-NoDerivs 4.0 International License (CC BY-NC-ND 4.0), which permits the noncommercial replication and distribution of the article with the strict proviso that no changes or edits are made and the original work is properly cited (including links to both the formal publication through the relevant DOI and the license). See: https://creativecommons.org/licenses/by-nc-nd/4.0/.

\section{References}

1. Bray F, Ferlay J, Soerjomataram I, et al. Global cancer statistics 2018: GLOBOCAN estimates of incidence and mortality worldwide for 36 cancers in 185 countries. CA Cancer J Clin 2018;68:394-424.

2. Network. N.C.C. Bladder Cancer (Version 4.2019). 2019; Available online: https://www.nccn.org/professionals/ physician_gls/pdf/bladder.pdf

3. Alfred Witjes J, Lebret T, Comperat EM, et al. Updated 2016 EAU Guidelines on Muscle-invasive and Metastatic Bladder Cancer. Eur Urol 2017;71:462-75.

4. Hermans TJN, Fransen van de Putte EE, Horenblas S, et al. Perioperative treatment and radical cystectomy for bladder cancer--a population based trend analysis of 10,338 patients in the Netherlands. Eur J Cancer 2016;54:18-26.

5. Hsu FS, Su CH, Huang KH. A Comprehensive Review of US FDA-Approved Immune Checkpoint Inhibitors in Urothelial Carcinoma. J Immunol Res 2017;2017:6940546.

6. Grossman HB, Natale RB, Tangen CM, et al. Neoadjuvant chemotherapy plus cystectomy compared with cystectomy 
alone for locally advanced bladder cancer. N Engl J Med 2003;349:859-66.

7. Griffiths G, Hall R, Sylvester R, et al. International phase III trial assessing neoadjuvant cisplatin, methotrexate, and vinblastine chemotherapy for muscle-invasive bladder cancer: long-term results of the BA06 30894 trial. J Clin Oncol 2011;29:2171-7.

8. Yin M, Joshi M, Meijer RP, et al. Neoadjuvant Chemotherapy for Muscle-Invasive Bladder Cancer: A Systematic Review and Two-Step Meta-Analysis. Oncologist 2016;21:708-15.

9. Burger M, Mulders P, Witjes W. Use of neoadjuvant chemotherapy for muscle-invasive bladder cancer is low among major European centres: results of a feasibility questionnaire. Eur Urol 2012;61:1070-1.

10. Balar AV, Galsky MD, Rosenberg JE, et al. Atezolizumab as first-line treatment in cisplatin-ineligible patients with locally advanced and metastatic urothelial carcinoma: a single-arm, multicentre, phase 2 trial. Lancet 2017;389:67-76.

11. Balar AV, Castellano D, O'Donnell PH, et al. First-line pembrolizumab in cisplatin-ineligible patients with locally advanced and unresectable or metastatic urothelial cancer (KEYNOTE-052): a multicentre, single-arm, phase 2 study. Lancet Oncol 2017;18:1483-92.

12. Rosenberg JE, Hoffman-Censits J, Powles T, et al. Atezolizumab in patients with locally advanced and metastatic urothelial carcinoma who have progressed following treatment with platinum-based chemotherapy: a single-arm, multicentre, phase 2 trial. Lancet 2016;387:1909-20.

13. Bellmunt J, de Wit R, Vaughn DJ, et al. Pembrolizumab as Second-Line Therapy for Advanced Urothelial Carcinoma. N Engl J Med 2017;376:1015-26.

14. Sharma P, Retz M, Siefker-Radtke A, et al. Nivolumab in metastatic urothelial carcinoma after platinum therapy (CheckMate 275): a multicentre, single-arm, phase 2 trial. Lancet Oncol 2017;18:312-22.

15. Powles T, O'Donnell PH, Massard C, et al. Efficacy and Safety of Durvalumab in Locally Advanced or Metastatic Urothelial Carcinoma: Updated Results From a Phase 1/2 Open-label Study. JAMA Oncol 2017;3:e172411.

16. Patel MR, Ellerton J, Infante JR, et al. Avelumab in metastatic urothelial carcinoma after platinum failure (JAVELIN Solid Tumor): pooled results from two expansion cohorts of an open-label, phase 1 trial. Lancet Oncol 2018;19:51-64.

17. Necchi A, Anichini A, Raggi D, et al. Pembrolizumab as Neoadjuvant Therapy Before Radical Cystectomy in Patients With Muscle-Invasive Urothelial Bladder Carcinoma (PURE-01): An Open-Label, Single-Arm, Phase II Study. J Clin Oncol 2018;36:3353-60.

18. Parekh DJ, Reis IM, Castle EP, et al. Robot-assisted radical cystectomy versus open radical cystectomy in patients with bladder cancer (RAZOR): an open-label, randomised, phase 3, non-inferiority trial. Lancet 2018;391:2525-36.

19. Powles T, Rodriguez-Vida A, Duran I, et al. A phase II study investigating the safety and efficacy of neoadjuvant atezolizumab in muscle invasive bladder cancer (ABACUS). J Clin Oncol 2018;36:abstr 4506.

20. Hoimes CJ, Albany C, Hoffman-Censits J, et al. LBA33A phase Ib/II study of neoadjuvant pembrolizumab (pembro) and chemotherapy for locally advanced urothelial cancer (UC). Ann Oncol 2018;29.

21. Gao J, Siefker-Radtke AO, Navai N, et al. A pilot presurgical study evaluating anti-PD-L1 durvalumab (durva) plus anti-CTLA-4 tremelimumab (treme) in patients (pts) with high-risk muscle-invasive bladder carcinoma (MIBC) who are ineligible for cisplatinbased neoadjuvant chemotherapy (NAC). J Clin Oncol 2019;37:abstr 4551.

22. Rodriguez-Moreno JF, Sevillano E, Fenor M, et al. Impact of the combination of durvalumab (MEDI4736) plus olaparib (AZD2281) administered prior to surgery in the molecular profile of resectable urothelial bladder cancer: NEODURVARIB Trial. J Clin Oncol 2019;37:TPS503-TPS.

23. Paz-Ares LG, Solsona E, Esteban E, et al. Randomized phase III trial comparing adjuvant paclitaxel/gemcitabine/ cisplatin (PGC) to observation in patients with resected invasive bladder cancer: Results of the Spanish Oncology Genitourinary Group (SOGUG) 99/01 study. J Clin Oncol 2010;28:LBA4518-LBA.

24. Cognetti F, Ruggeri EM, Felici A, et al. Adjuvant chemotherapy with cisplatin and gemcitabine versus chemotherapy at relapse in patients with muscle-invasive bladder cancer submitted to radical cystectomy: an Italian, multicenter, randomized phase III trial. Ann Oncol 2012;23:695-700.

25. Sternberg CN, Skoneczna I, Kerst JM, et al. Immediate versus deferred chemotherapy after radical cystectomy in patients with pT3-pT4 or $\mathrm{N}+\mathrm{M} 0$ urothelial carcinoma of the bladder (EORTC 30994): an intergroup, open-label, randomised phase 3 trial. Lancet Oncol 2015;16:76-86.

26. Steenbruggen TG, van Ramshorst MS, Kok M, et al. Neoadjuvant Therapy for Breast Cancer: 
Established Concepts and Emerging Strategies. Drugs 2017;77:1313-36.

27. Roghmann F, Trinh QD, Braun K, et al. Standardized assessment of complications in a contemporary series of European patients undergoing radical cystectomy. Int $\mathbf{J}$ Urol 2014;21:143-9.

28. Shabsigh A, Korets R, Vora KC, et al. Defining early morbidity of radical cystectomy for patients with bladder cancer using a standardized reporting methodology. Eur Urol 2009;55:164-74.

29. Melero I, Berraondo P, Rodriguez-Ruiz ME, et al. Making the Most of Cancer Surgery with Neoadjuvant Immunotherapy. Cancer Discov 2016;6:1312-4.

30. Liu J, Blake SJ, Yong MC, et al. Improved Efficacy of Neoadjuvant Compared to Adjuvant Immunotherapy to Eradicate Metastatic Disease. Cancer Discov 2016;6:1382-99.

31. Choueiri TK, Jacobus S, Bellmunt J, et al. Neoadjuvant dose-dense methotrexate, vinblastine, doxorubicin, and cisplatin with pegfilgrastim support in muscle-invasive urothelial cancer: pathologic, radiologic, and biomarker correlates. J Clin Oncol 2014;32:1889-94.

32. Plimack ER, Hoffman-Censits JH, Viterbo R, et al. Accelerated methotrexate, vinblastine, doxorubicin, and cisplatin is safe, effective, and efficient neoadjuvant treatment for muscle-invasive bladder cancer: results of a multicenter phase II study with molecular correlates of response and toxicity. J Clin Oncol 2014;32:1895-901.

33. Iyer G, Balar AV, Milowsky MI, et al. Multicenter Prospective Phase II Trial of Neoadjuvant Dose-Dense

Cite this article as: Kim IH, Lee HJ. Perioperative immunotherapy for muscle-invasive bladder cancer. Transl Androl Urol 2020;9(6):2976-2985. doi: 10.21037/tau.2019.11.31
Gemcitabine Plus Cisplatin in Patients With MuscleInvasive Bladder Cancer. J Clin Oncol 2018;36:1949-56.

34. Powles T, Duran I, van der Heijden MS, et al. Atezolizumab versus chemotherapy in patients with platinum-treated locally advanced or metastatic urothelial carcinoma (IMvigor211): a multicentre, open-label, phase 3 randomised controlled trial. Lancet 2018;391:748-57.

35. Sharma $P$, Callahan MK, Bono $P$, et al. Nivolumab monotherapy in recurrent metastatic urothelial carcinoma (CheckMate 032): a multicentre, open-label, two-stage, multi-arm, phase 1/2 trial. Lancet Oncol 2016;17:1590-8.

36. Kim HS, Seo HK. Immune checkpoint inhibitors for urothelial carcinoma. Investig Clin Urol 2018;59:285-96.

37. Garon EB, Rizvi NA, Hui R, et al. Pembrolizumab for the treatment of non-small-cell lung cancer. N Engl J Med 2015;372:2018-28.

38. Colli LM, Machiela MJ, Myers TA, et al. Burden of Nonsynonymous Mutations among TCGA Cancers and Candidate Immune Checkpoint Inhibitor Responses. Cancer Res 2016;76:3767-72.

39. Le DT, Durham JN, Smith KN, et al. Mismatch repair deficiency predicts response of solid tumors to PD-1 blockade. Science 2017;357:409-13.

40. McGranahan N, Furness AJ, Rosenthal R, et al. Clonal neoantigens elicit $\mathrm{T}$ cell immunoreactivity and sensitivity to immune checkpoint blockade. Science 2016;351:1463-9.

41. Cancer Genome Atlas Research Network. Comprehensive molecular characterization of urothelial bladder carcinoma. Nature 2014;507:315-22. 